\title{
Atomic Configuration of Planar Defects in Multiferroic Ca-doped $\mathrm{BiFeO}_{3}$ Films
}

\author{
M.D. Rossell,* Q.M. Ramasse,** R. Erni,*** C.-H. Yang,**** and R. Ramesh***** \\ * Laboratory for Multifunctional Materials, Department of Materials, ETH Zürich, 8093 Zürich, \\ Switzerland \\ ** SuperSTEM Laboratory, STFC Daresbury, Warrington WA4 4AD, United Kingdom \\ *** Electron Microscopy Center, Swiss Federal Laboratories for Materials Testing and Research \\ (Empa), 8600 Dübendorf, Switzerland \\ **** Department of Physics, Korea Advanced Institute of Science and Technology (KAIST), \\ Yuseong-gu, Daejeon 305-701, Republic of Korea \\ ***** Department of Physics, University of California, Berkeley, California 94720
}

The multiferroic $\mathrm{BiFeO}_{3}$ (BFO) offers great potential applications in the nonvolatile ferroelectric random access memory and magnetic data storage due to its large room-temperature spontaneous polarization $\left(\sim 90 \mu \mathrm{C} / \mathrm{cm}^{2}\right)$ and the possibility of coupling between the ferroelectric polarization and antiferromagnetism. However, one of the main drawbacks that hamper the application of BFO is its high leakage current which is related to the defects in the material, in particular, oxygen vacancies. Efforts are currently being made to reduce the oxygen vacancies by using different deposition oxygen pressure, post-annealing the BFO in oxygen and doping BFO with higher valence ions.

In this work we explore the mechanism of oxygen-vacancy formation in divalent-ion-calcium-doped BFO films by annular dark-field (ADF) and bright-field (BF) aberration-corrected imaging performed in scanning transmission electron microscopy (STEM) mode.

Figure 1 shows an ADF-STEM micrograph of a 20\% Ca-doped BFO film grown on a (001) $\mathrm{SrTiO}_{3}$ substrate using pulsed laser deposition [1]. The film is characterized by the presence of single perovskite-type dark layers appearing periodically every 7 or 8 perovskite blocks along the out-ofplane direction, as confirmed by the occurrence of an incommensurate modulation along the [001] direction in the corresponding power spectrum. Besides, the presence of reflections outlined by white arrows in the power spectrum indicate that the projected potentials at the lattice points separated by $b$ $(\sim 0.39 \mathrm{~nm})$ are not equal, but they are equal every second perovskite unit cell. By using BF and ADF-STEM imaging we investigate the origin of this "doubling" of period.

The ADF-STEM image in Figure 2a clearly shows an increasing concentration of Bi along the film growth direction between consecutive dark layers. At the bottom of each planar defect a rock-salt ordered arrangement of $\mathrm{Bi} / \mathrm{Ca}$ cations is adopted and promotes the formation of oxygen vacancies. A brownmillerite-like intra-plane ordering of oxygen vacancies is revealed in the BF-STEM image in Figure $2 \mathrm{~b}$. Besides, a small concentration of ordered vacancies is also present in the layer located below the brownmillerite-type layers. Thus, the larger $\mathrm{Ca}$ cations retain twelve-fold oxygen coordination, while removal of oxygen atoms in each second position along the $b$ direction would leave the $\mathrm{Bi}$ smaller cations in ten-fold oxygen coordination. These ten-fold oxygen coordinated atoms would be slightly displaced from their original positions as they move away from the oxygen vacancies [2]. 
References

[1] C.-H. Yang et al., Nature Materials 8 (2009) 485.

[2] Part of this work was performed at NCEM, which is supported by the Office of Science, Office of Basic Energy Sciences of the U.S. Department of Energy under Contract No. DE-AC02$05 \mathrm{CH} 11231$.
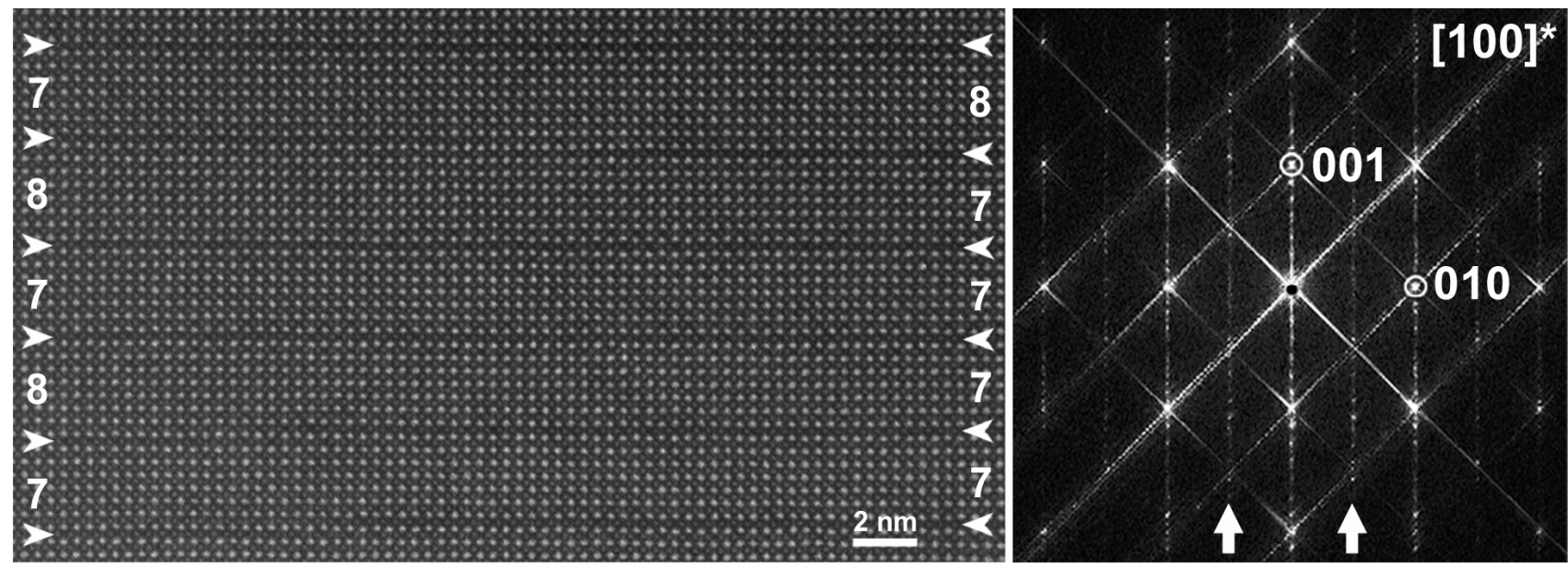

FIG. 1. Atomic configuration of a $20 \%$ Ca-doped $\mathrm{BiFeO}_{3}$ film. Left: ADF-STEM unprocessed micrograph. The growth direction is from bottom to top. White arrowheads indicate the position of the dark layers at each side of the image. Right: Corresponding power spectrum indexed assuming a pseudo-tetragonal unit cell [1].
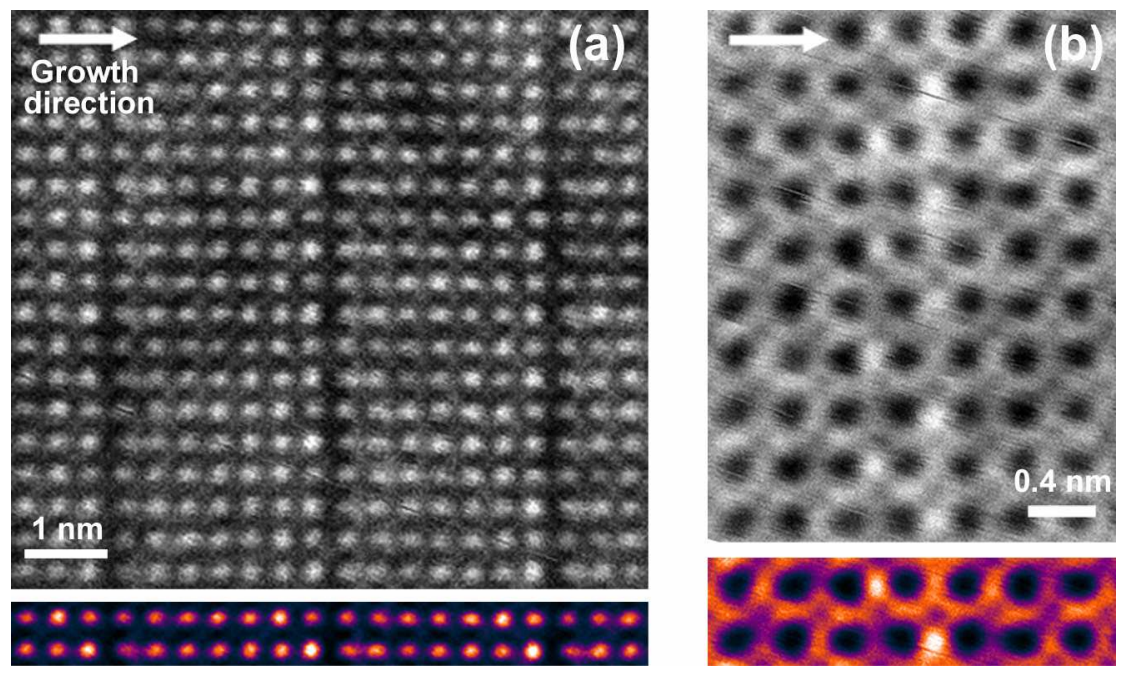

FIG. 2. (a) ADF-STEM image of three dark layers showing the arrangement of the A-type $(\mathrm{A}=\mathrm{Bi}$, $\mathrm{Ca}$ ) atomic columns. The ADF image shows strong contrast for the heavy Bi/Ca atomic columns and poor contrast for the lighter $\mathrm{Fe}$ atoms. The $\mathrm{Ca}$ ions have a tendency to occupy the A-type positions in a random fashion at the right side of each dark layer, but a rock-salt ordered arrangement is adopted at the left side of the dark layers. (b) BF-STEM image of a planar defect showing the intra-plane ordering of oxygen vacancies. White dots correspond to oxygen atom columns (poor contrast) and oxygen depleted columns (strong contrast). The colored images show the result of averaging over the entire images. 